\title{
Rectus sheath hematoma presenting as an abdominal mass
}

\author{
Kinagabran Sivananthan ${ }^{1}$, Karthigesu Aimanan ${ }^{2}$, Senthilvasan Kanthasamy ${ }^{2}$, Azlanudin Azman ${ }^{1}$, Ramesh Thangaratnam ${ }^{2}$ and Chew Loon \\ Guan $^{2}$ \\ ${ }^{1}$ Department of Surgery, National University of Malaysia, Cheras, Malaysia \\ ${ }^{2}$ Department of Surgery, Hospital Serdang, Selangor, Malaysia
}

\begin{abstract}
Pathology arising from the abdominal wall is frequently overlooked as the cause for an abdominal mass. Although clinical presentation of the rectus muscle hematoma is well documented, the diagnosis remains a challenge. Hematoma within the rectus sheath produces a painful, tender swelling that can mimic an intraperitoneal mass. This is a case report of an elderly male presenting with rectus sheath hematoma after bouts of prolonged cough.
\end{abstract}

\section{Introduction}

Rectus sheath hematoma is a rare clinical condition and often misdiagnosed cause of abdominal mass. It is a well-documented complication of abdominal trauma, surgery and excessive strain on abdominal musculature. This is a case report of rectus sheath hematoma in which the clinical features and ultrasound findings favored intraperitoneal mass. However, CT scan well delineates the mass and pointed towards the diagnosis of rectus sheath hematoma.

\section{Case Presentation}

88 years old Chinese male with an underlying cerebrovascular accident since 15 years ago, hypertension and COAD presented to the emergency care with a complaint of abdominal swelling for one month. He reported that the abdominal swelling was associated with discomfort, loss of weight and loss of appetite but denied the change in bowel habits and abdominal trauma. He was on aspirin, clopidogrel, and neulin. Examination revealed a firm swelling over the right lumbar region with mild tenderness and guarding. Bowel sounds were normal. Blood results showed leucocytosis, Hemoglobin of 10.7 and INR 1.1. The patient was provisionally diagnosed to have a colonic tumor and was admitted for colonoscopy. However, during admission, noted patient developed shortness of breath with a drop in oxygen saturation. Auscultation of the lungs revealed generalized crepitations and rhonchi. Chest $\mathrm{x}$-ray showed pneumonic patches with right upper lobe consolidation. Colonoscope had to be abandoned due to the acute episode of breathlessness, and we proceeded with ultrasound of the abdomen which showed heterogenous mass at the right lumbar region, originating from the bowel. Subsequently contrasted CT scan of the abdomen confirmed the swelling and demonstrated thickened right rectus abdominis muscle with an ill-defined fluid collection (Figure 1). This finding was consistent with rectus sheath hematoma. On further history from family members revealed, the patient had poor control of COAD with a history of prolonged cough for the last two months. Therefore the final diagnosis is rectus sheath hematoma secondary to protracted cough with exacerbation of COAD secondary to pneumonia. The patient was co-managed with the medical team and was started on antibiotics, and regular nebulization. His symptoms were improved over the next few days, abdominal wall swelling reduced and was

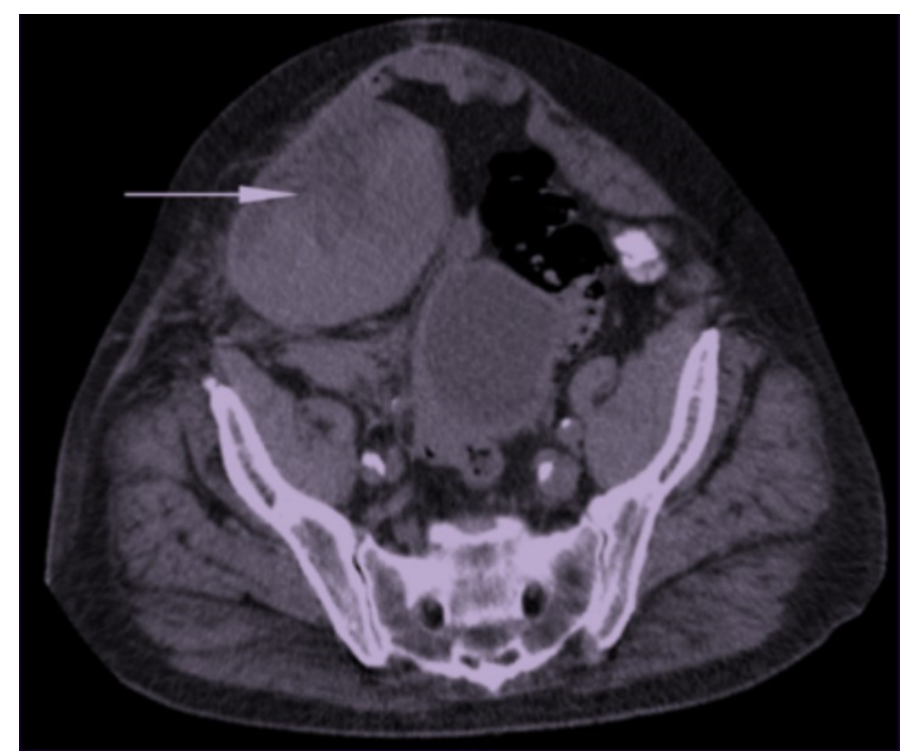

Figure 1. Hematoma along the right abdominal wall involving rectus sheath

discharged well. The patient was given follow up under respiratory care unit to optimize his COAD.

\section{Discussion}

Rectus sheath hematoma is a known complication of abdominal trauma, surgery and excessive strain on the abdominal musculature. Due to the anatomy of the abdominal wall below the arcuate line, rupture of epigastric vessels or muscle within rectus sheath causes a hematoma mimicking acute abdomen or abdominal mass. Rectus sheath hematoma commonly presents with abdominal pain, swelling, and a decrease in hemoglobin, abdominal wall ecchymosis, nausea,

${ }^{\star}$ Correspondence to: Karthigesu Aimanan M.S (UKM), General Surgeon, Department of Surgery, Hospital Serdang, Selangor, Malaysia, E-mail: karthi abim@yahoo.com

Received: June 09, 2018; Accepted: June 17, 2018; Published: June 25, 2018 
vomiting and tachycardia [1]. Physical examination reveals tenderness and swelling in the abdominal wall. Generally, the abdominal mass in RSH does not cross the midline. Fothergill's sign and Carnett sign are positive in rectus sheath hematoma, and helps to differentiate this condition from intraabdominal pathologies [2]. Fothergill' sign is positive when the haematoma within the rectus sheath produces a mass that does not cross the midline and remains palpable when the patient tenses his rectus muscle by touching his chest using his chin [3]. Similarly, tenderness remains the same or increases with head raising and is referred to as Carnett's sign [4]. Ultrasonography is a useful initial test to differentiate intraperitoneal lesions from extraperitoneal lesions, however the interpretation is subjected to operators experiences [5]. Therefore, non-contrast CT scan is more accurate and is the diagnostic modality of choice [6]. According to CT scan, type I hematomas are mild, and the hematoma occurs within the muscle with an increase in muscle length and does not require hospitalization [7]. Type II hematomas are moderate, the hematoma is within the muscle but bleeding occurs into the space between transversalis fascia and the muscle. Type III hematomas are severe and located between transversalis fascia and the muscle, anterior to the peritoneum and urinary bladder. Conservative treatment is the mainstay of management in hemodynamically stable patients with non-expanding hematoma however with the failure of conservative treatment, a surgical approach can be chosen, but the mortality rates of surgery for rectus sheath hematoma is high [8].

\section{Conclusion}

Rectus sheath hematoma can frequently mimic other common abdominal emergencies leading to delay in diagnosis. Prompt history taking with careful physical examination and appropriate imaging studies in which CT scan seems to be the most appropriate choice of imaging helps to avoid unnecessary laparotomies and decrease mortality and morbidity.

\section{References}

1. Siu WT, Yau KK, Cheung HY, Law BK, Tang CN, et al. (2003) Spontaneous rectus sheath hematoma. Can J Surg 46: 390.

2. Osinbowale O, Bartholomew JR (2008) Rectus sheath hematoma. Vasc Med 13: 275279. [Crossref]

3. Karabulut Z, Abci I, Lakadamyali H, Gebedek O (2006) [A case of rectus sheath hematoma]. Ulus Travma Acil Cerrahi Derg 12: 76-78. [Crossref]

4. Costello J, Wright J (2005) Rectus sheath haematoma: 'a diagnostic dilemma?. Emerg Med J 22: 523-524. [Crossref]

5. Thomson H, Francis DM (1977) Abdominal-wall tenderness: A useful sign in the acute abdomen. Lancet 2: 1053-1054. [Crossref]

6. Hecker RB, Bradshaw WH, Pinkerton SF (1990) Rectus sheath hematoma: report of a case. Tex Med 86: 68-70. [crossref]

7. Moreno Gallego A, Aguayo JL, Flores B, Soria T, Hernández Q, et al. (1997) Ultrasonography and computed tomography reduce unnecessary surgery in abdominal rectus sheath hematoma. Br J Surg 84: 1295-1297. [Crossref]

8. Luhmann A, Williams EV (2006) Rectus sheath hematoma: a series of unfortunate events. World J Surg 30: 2050-2055. [Crossref]

Copyright: $₫ 2018$ Sivananthan K. This is an open-access article distributed under the terms of the Creative Commons Attribution License, which permits unrestricted use, distribution, and reproduction in any medium, provided the original author and source are credited. 